Article

\title{
Element-Specific Magnetization Dynamics of Complex Magnetic Systems Probed by Ultrafast Magneto-Optical Spectroscopy
}

\author{
Clemens von Korff Schmising ${ }^{1, *}{ }^{\mathbb{C}}$, Felix Willems ${ }^{1}{ }^{\mathbb{D}}$, Sangeeta Sharma ${ }^{1}$, Kelvin Yao ${ }^{1}$,

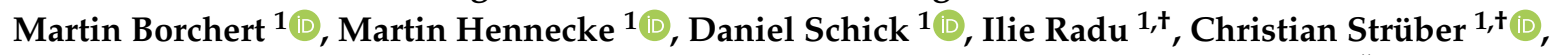

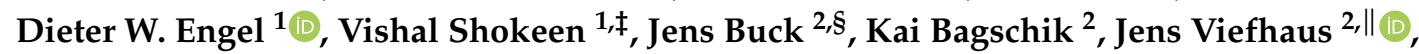 \\ Gregor Hartmann ${ }^{2, \|}$, Bastian Manschwetus ${ }^{2}$, Soeren Grunewald ${ }^{2}$, Stefan Düsterer ${ }^{2}$ (D), \\ Emmanuelle Jal $^{3} \mathbb{D}$, Boris Vodungbo ${ }^{3} \mathbb{D}$, Jan Lüning ${ }^{3, \|}$ and Stefan Eisebitt ${ }^{1,4}(\mathbb{D}$ \\ 1 Max-Born-Institut, Max-Born-Strasse 2A, 12489 Berlin, Germany; felix.willems@gmx.de (F.W.); \\ sharma@mbi-berlin.de (S.S.); yao@mbi-berlin.de (K.Y.); borchert@mbi-berlin.de (M.B.); \\ hennecke@mbi-berlin.de (M.H.); schick@mbi-berlin.de (D.S.); radu@mbi-berlin.de (I.R.); \\ cstrueber@zedat.fu-berlin.de (C.S.); engel@mbi-berlin.de (D.W.E.); vishalshokeen001@gmail.com (V.S.); \\ eisebitt@mbi-berlin.de (S.E.) \\ 2 Deutsches Elektronen-Synchrotron (DESY), Notkestrasse 85, 22607 Hamburg, Germany; \\ jens.buck@desy.de (J.B.); kai.bagschik@desy.de (K.B.); jens.viefhaus@helmholtz-berlin.de (J.V.); \\ gregor.hartmann@helmholtz-berlin.de (G.H.); bastian.manschwetus@desy.de (B.M.); \\ soeren.grunewald@desy.de (S.G.); stefan.duesterer@desy.de (S.D.) \\ 3 Laboratoire de Chimie Physique-Matière et Rayonnement, LCPMR, Sorbonne Université, CNRS, \\ 75005 Paris, France; emmanuelle.jal@sorbonne-universite.fr (E.J.); boris.vodungbo@sorbonne-universite.fr \\ (B.V.); jan.luning@helmholtz-berlin.de (J.L.) \\ 4 Institut für Optik und Atomare Physik, Technische Universitat Berlin, 10623 Berlin, Germany \\ * Correspondence: korff@mbi-berlin.de \\ + Current address: Department of Physics, Free University Berlin, Arnimallee 14, 14195 Berlin, Germany. \\ $\ddagger$ Current address: Department of Physics and Astronomy, Uppsala University, Uppsala, Sweden. \\ $\S$ Current address: Department of Physics, Universität Kiel, Kiel, Germany. \\ || Current address: Helmholtz-Zentrum Berlin für Materialien und Energie, Albert-Einstein-Straße 15, \\ 12489 Berlin, Germany.
}

Received: 23 September 2020; Accepted: 21 October 2020; Published: 28 October 2020

\begin{abstract}
The vision to manipulate and control magnetism with light is driven on the one hand by fundamental questions of direct and indirect photon-spin interactions, and on the other hand by the necessity to cope with ever growing data volumes, requiring radically new approaches on how to write, read and process information. Here, we present two complementary experimental geometries to access the element-specific magnetization dynamics of complex magnetic systems via ultrafast magneto-optical spectroscopy in the extreme ultraviolet spectral range. First, we employ linearly polarized radiation of a free electron laser facility to demonstrate decoupled dynamics of the two sublattices of an FeGd alloy, a prerequisite for all-optical magnetization switching. Second, we use circularly polarized radiation generated in a laboratory-based high harmonic generation setup to show optical inter-site spin transfer in a CoPt alloy, a mechanism which only very recently has been predicted to mediate ultrafast metamagnetic phase transitions.
\end{abstract}

Keywords: ultrafast demagnetization dynamics; transient absorption spectroscopy; free electron laser; high harmonic generation 


\section{Introduction}

The absorption of a femtosecond laser pulse by a thin magnetic film induces a non-equilibrium and spin-dependent electron distribution that triggers a cascade of microscopic processes and causes a very rapid change in the magnetization [1,2]. Spin-orbit coupling mediates spin-flips in scattering events and leads to a local loss of the magnetization [3]. Magnon generation quenches the long-range magnetic order [4-6], and the spin-dependent transport properties of excited electrons trigger spin currents and redistribute spin angular momentum within the sample $[7,8]$.

One of the most fascinating observations in the field of femtomagnetism is all-optical switching, which was first discovered in the rare earth-transition metal alloy, FeGd [9]. Upon optical excitation, the antiferromagnetically coupled $\mathrm{Gd}$ and Fe sites can be promoted into a transient ferromagnetic state, which mediates a reversal of macroscopic magnetization [10,11]. Microscopically, this is understood by a transient suppression of the inter-atomic exchange interaction due to ultrafast heating of the electrons, enabling an independent response of the rare earth and transition metal sublattices. Upon cooling, angular momentum exchange breaks the symmetry of the system and leads to magnetization switching. Because of the low specific heat of electrons, all-optical switching is inherently energy efficient with obvious potential for applications.

Very recently, a completely new mechanism to manipulate the magnetic structure has been put forward theoretically: laser pulse excitation induces an optical inter-site spin transfer (OISTR) in multi-element magnetic systems [12]. This spin manipulation is of a purely optical nature and is therefore one of the fastest ways to manipulate spins by light. The authors of this theoretical study also predicted that the spin-selective charge transfer between distinct sites can lead to a metamagnetic phase transition, switching the magnetic order from an antiferromagnetic to a transient ferromagnetic alignment. In the last two years, a number of experiments demonstrated signatures of OISTR, both in the optical $[13,14]$ as well as in the extreme ultraviolet (XUV) [15-18] spectral range. The latter experiments were carried out with high harmonic generation (HHG) sources and exploited the element specificity of resonant core-to-valence state transitions. Here, the sensitivity to the local magnetic moment can be easily explained by a two step model [19]: if circularly polarized photons are absorbed by core level electrons with finite spin-orbit interaction the resulting photoelectrons become spin-polarized. Then, the helicity-dependent absorption strength in the material depends on the spin imbalance of the ferromagnet, with different numbers of available states for majority and minority electrons in the exchange-split valence band. Therefore, the observable, calculated as the difference of the transmitted light intensity for opposite circular polarization, is directly proportional to the magnetization and is called magnetic circular dichroism (MCD). More generally, the optical response around such a resonant transition in magnetic materials can be parameterized by a complex magneto-optical function.

While time resolved MCD measurements have been pioneered in the soft $\mathrm{X}$-ray range at the L-edges of transition metals, in particular, at the femto-slicing beamline at BESSY, HZB, Germany [20], in the last decade a lot of effort has been devoted to transferring resonant probing techniques of magnetization to more shallow resonances in the XUV spectral range, both at free electron laser (FEL) facilities [21] and at laboratory-based HHG sources in reflection [22,23] or transmission geometry [24]. Research has focused on tracking laser-driven, ultrafast spin transport either using the transverse magneto-optical Kerr effect $[25,26]$ or by exploiting the short wavelength of the XUV radiation in time-resolved small angle X-ray scattering experiments [27-30] as well as via ultrafast coherent imaging experiments [31]. Furthermore, the great potential of probing magnetization with resonant XUV radiation was convincingly demonstrated in a series of experiments, which showed that transient spectra can provide fingerprint signatures of microscopic processes responsible for ultrafast demagnetization, in particular differentiating Heisenberg type delocalized magnon or Stoner type spin-flip excitations [32-34].

In this contribution, we present two complementary experiments dedicated to an element-specific view of femtomagnetism based on ultrafast XUV spectroscopy of multi-component magnetic systems 
performed at the FEL facility, FLASH, DESY, Hamburg and at a HHG beamline at the Max Born Insitute, Berlin. At the FEL facility, we exploit the dispersive (real) part of the magneto-optical functions in a resonant Faraday rotation geometry to study the ultrafast response of an FeGd alloy and reveal decoupled dynamics of the two sublattices Gd and Fe. In the experiment using HHG radiation, we compare the ultrafast response of a Co film and a CoPt alloy by measuring the absorptive (imaginary) part of the magneto-optical functions in an MCD experiment. Here, we were able to demonstrate that OISTR between $\mathrm{Pt}$ and $\mathrm{Co}$ atoms in the two-component $\mathrm{CoPt}$ alloy dominates the early time dynamics and leads to a faster and more efficient demagnetization.

\section{Results and Discussions}

\subsection{Static Magneto-Optical Functions}

Before we discuss the time resolved experiments, we briefly review details on the complex magneto-optical function in the XUV spectral range, which allows us to probe the element-specific response of multi-component magnetic systems.

Linearly polarized electromagnetic radiation can be decomposed into two circularly polarized waves with opposite helicity and equal amplitude. Its propagation through a magnetized material is then characterized by the complex index of refraction:

$$
n_{ \pm}(E)=1-(\delta(E) \pm \Delta \delta(E))+i(\beta(E) \pm \Delta \beta(E)) .
$$

$\delta(E)$ and $\beta(E)$ are the dispersive and absorptive parts of the electro-optical functions valid at the photon energy, $E$, and describe the interaction with non-magnetic materials. The effect of the magnetization is given by the magneto-optical functions $\Delta \beta(E)$ and $\Delta \delta(E)$. The positive and negative sign refers to either parallel or antiparallel alignment of the magnetization with respect to the wave vector of the incident radiation. Equation (1) directly implies that the element specific magnetization can be accessed either via the dispersive or absorptive part of the magneto-optical function, leading to two possible experimental configurations in a transmission geometry, MCD and Faraday rotation.

The absorptive magnetic contribution, $\Delta \beta$, leads to a difference in transmitted intensity, $I_{ \pm}$, between left and right circularly polarized light and is generally referred to as MCD. It is defined for a sample with thickness, $d$, as:

$$
\Delta \beta(E)=-\frac{\hbar c}{4 E d} \ln \left(\frac{I_{+}}{I_{-}}\right) .
$$

The dispersive magnetic contribution, $\Delta \delta$, leads to a difference in the phase of the two oppositely polarized modes and rotates the plane of polarization of the incoming radiation. The corresponding rotation angle, $\theta_{F}$, is called Faraday angle and can be expressed as:

$$
\theta_{F}(E)=\frac{E d}{\hbar c} \Delta \delta
$$

This equation is valid for a normal incident geometry and an out-of-plane magnetization, such that refraction of the radiation at the sample interfaces does not need to be considered [24,35-37].

In Figure 1, we show the magneto-optical functions of an $\mathrm{Fe}_{26} \mathrm{Gd}_{74}(15 \mathrm{~nm}$ thickness) alloy at the Gd $N_{4,5}$ resonance $(4 d \rightarrow 4 f)$ around $150 \mathrm{eV}$ and at the Fe $M_{2,3}(3 p \rightarrow 3 d)$ resonance around $55 \mathrm{eV}$. The measurements were performed at the UE112 beamline of the BESSY II synchrotron light source, HZB, Germany. We recorded $I_{ \pm}$and calculated the values of $\Delta \beta$ according to Equation (2). The corresponding dispersive part, $\Delta \delta$, is determined via the Kramers-Kronig relation. Note that, because the magneto-optical functions are equal to zero away from the resonance, the connecting real or imaginary part of the complex response function $n_{ \pm}(E)$ can be computed with high accuracy [38]. We also show the deduced Faraday rotation, which reaches a maximum of $\approx 0.36 \mathrm{deg} / \mathrm{nm}$ for $\mathrm{Gd}$ 
around $149 \mathrm{eV}$ and $\approx 0.07 \mathrm{deg} / \mathrm{nm}$ for Fe around $56 \mathrm{eV}$. Note that this is about an order of magnitude larger than in the visible spectral range.

Co exhibits a magneto-optical function around its $M_{2,3}$ resonance with a similar shape and magnitude compared to Fe: the absorptive part reaches a maximum of $\Delta \beta \approx-0.014$ at $60.3 \mathrm{eV}$. In a CoPt alloy, Co atoms induce a finite magnetic moment at their nearest neighbor Pt atoms with parallel aligned magnetic moments (cf. Figure 2e). In the spectral range between 45 and $72.5 \mathrm{eV}$, we can probe the local magnetization of $\mathrm{Pt}$ via the $\mathrm{Pt} \mathrm{O}_{2,3}$ and $\mathrm{Pt} N_{7}$ resonances at 51.7, 65.3 and $71.2 \mathrm{eV}$, respectively [24,39]. For more details on the magneto-optical functions of the transition metals, as well as their ab initio description in a static and transient state, we refer to our recent works Willems et al. [38] and Dewhurst et al. [40].
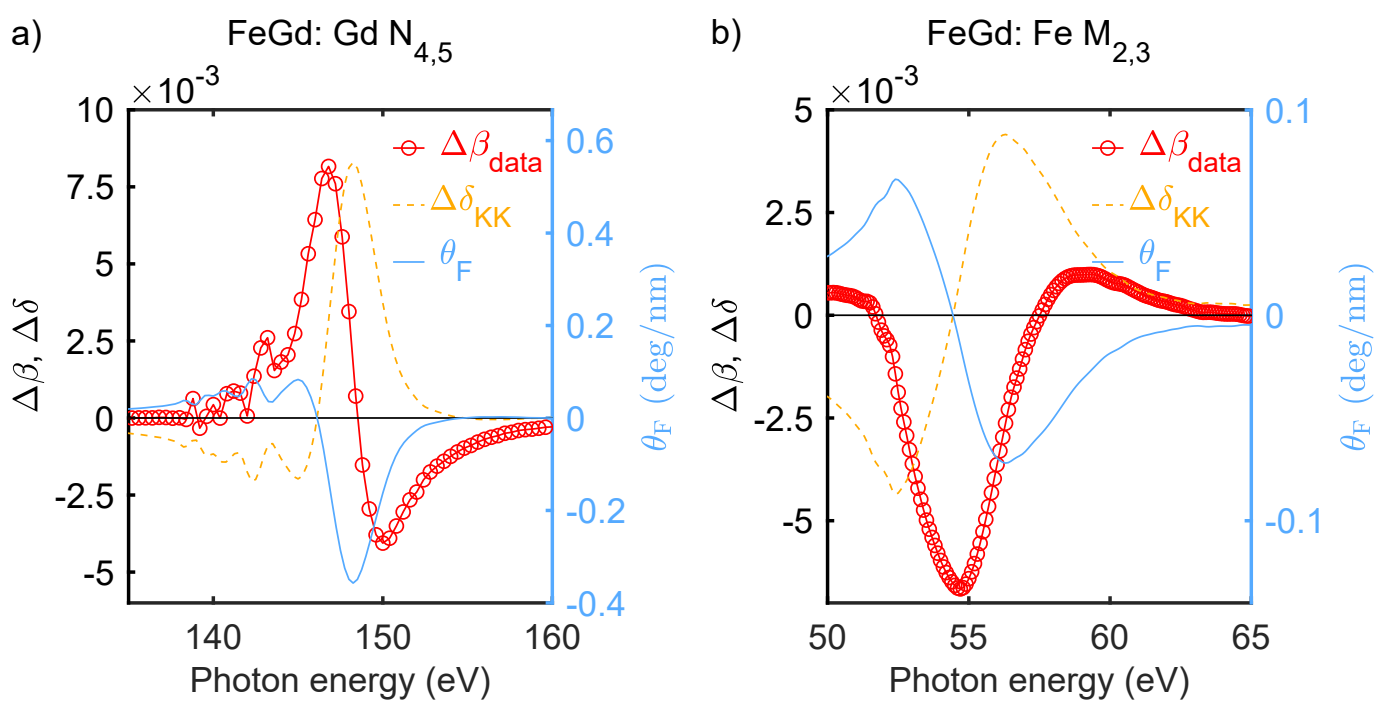

Figure 1. Magneto-optical functions, $\Delta \beta$ and $\Delta \delta$, of an $\mathrm{Fe}_{26} \mathrm{Gd}_{74}$ alloy (a) at the $\mathrm{Gd} N_{4,5}$ resonance around a photon energy of $E=150 \mathrm{eV}$ as well as (b) at the Fe $M_{2,3}$ resonance around a photon energy of $E=55 \mathrm{eV}$. The dispersive part, $\Delta \delta$, is retrieved by the Kramers-Kronig relation and $\theta_{F}$ is calculated according to Equation (3).

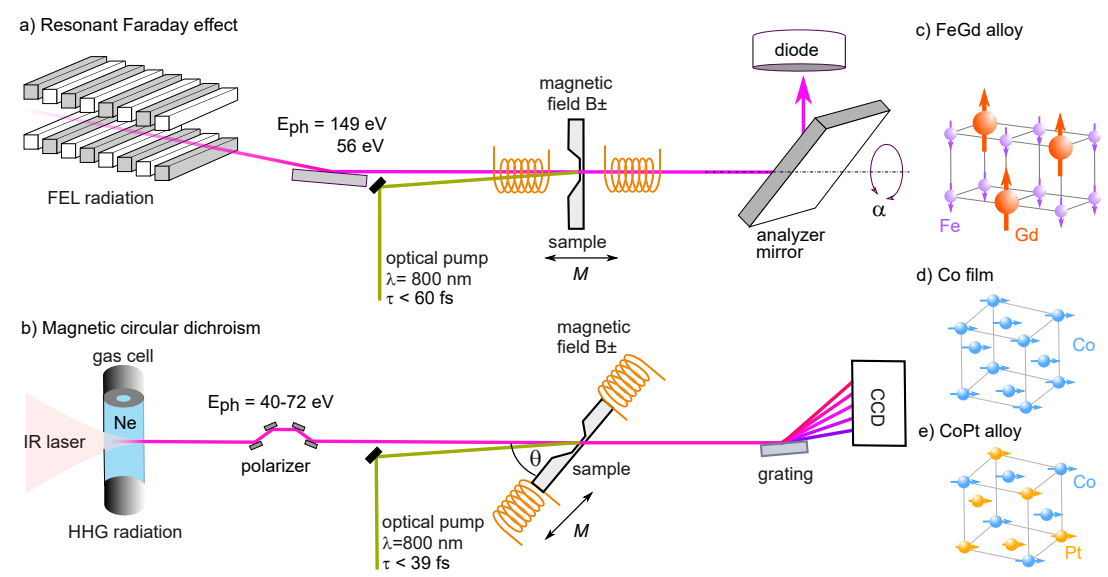

Figure 2. (a) Scheme of a Faraday setup using single color FEL radiation. A linearly polarized XUV pulse is transmitted through the sample and the Faraday rotation is detected by a Rabniovitch polarimeter. (b) Scheme of an MCD setup using HHG radiation. The multiple emission peaks of the radiation are circularly polarized by a reflective phase-shifter, transmitted through the magnetic sample and detected by a spectrometer. In both setups, we realized a pump-probe geometry to measure optical driven ultrafast magnetization dynamics. Schematic depiction of (c) an out-of-plane magnetized FeGd alloy and of an in-plane magnetized (d) Co and (e) CoPt film. 


\subsection{Time Resolved Faraday Rotation}

In this section, we present time resolved measurements based on the resonant Faraday Effect to demonstrate distinct ultrafast magnetization dynamics of the two magnetic sublattices of an FeGd ferrimagnetic alloy. The magnetic structure of the FeGd is schematically depicted in Figure $2 \mathrm{c}$ with Fe and Gd antiferromagnetically aligned in an out-of-plane direction. In panel (a) of Figure 2, we show the scheme of the experiment, as implemented at the beamline BL2 of the FEL facility FLASH, at DESY in Hamburg, Germany [41]. Linearly polarized pulses with a temporal duration between $50-80 \mathrm{fs}$ are tuned to a photon energy resonant with either the $\mathrm{Fe} M_{2,3}$ at $\approx 56 \mathrm{eV}$ or with the Gd $N_{4,5}$ edge at $\approx 149 \mathrm{eV}$ and impinge on the sample under normal incidence. The fluence of the XUV radiation is adjusted to remain below $1 \mathrm{~mJ} \mathrm{~cm}{ }^{-2}$ to avoid an influence of the probe pulses on the dynamics and to limit the risk of sample damage. The magnetization of $\mathrm{Fe}_{26} \mathrm{Gd}_{74}$ (15 nm thickness) can be set with an external magnetic field to point parallel $\left(B_{+}\right)$or antiparallel $\left(B_{-}\right)$to the wave vector of the probe pulses. After transmission through the magnetic sample, the polarization of the XUV pulses is analyzed by a Rabinovitch polarimeter [42], consisting of a mirror aligned at the Brewster angle and an XUV sensitive photo diode. For Gd, the analyzer is a multilayer mirror (OptixFab GmbH, Jena) which has been optimized for a large contrast between the reflectance of s- and p-polarized light centered at $150 \mathrm{eV}$ with a full width at half maximum of $10 \mathrm{eV}$. The reflectances, $R_{s, p}$, of the multilayer mirror were determined by a calibration measurement at the light source of the German national metrology institute, PTB, Berlin, Germany, using 99.3\% degree p-polarization. For Fe, we used a gold coated mirror. The polarizing power of the analyzer elements is defined as $P=\left(R_{s}-R_{p}\right) /\left(R_{s}+R_{p}\right)$ and listed with the respective reflectances in Table 1 . The mirror-detector assembly can be rotated around the beam axis by the azimuthal angle $\alpha$. Pump pulses centered at a wavelength of $800 \mathrm{~nm}$, with a pulse duration of $<60 \mathrm{fs}$ and with a fluence of $\approx 5 \mathrm{~mJ} \mathrm{~cm}^{-2}$ excite the sample in a collinear geometry to induce an ultrafast demagnetization. The time resolution is estimated to remain below $100 \mathrm{fs}$ in all experiments, taking into account the temporal jitter of arrival times between optical and XUV pulses. Measurements are performed in a repetitive pump-probe scheme by averaging 100 shots at a $10 \mathrm{~Hz}$ repetition rate for each rotation angle or time delay. All data are normalized shot-to-shot by the photon numbers recorded by the gas monitor detector of the FLASH photon diagnostics.

Table 1. Reflectances, $R_{s, p}$, and polarizing power, $P=\left(R_{s}-R_{p}\right) /\left(R_{s}+R_{p}\right)$, of the analyzer mirrors. The values for the ML mirror were determined at the Metrology Light source of PTB, Berlin, and the values for the Au mirror were calculated with a tabulated index of refraction at $56 \mathrm{eV}$ [43]. The Brewster angles are measured in a grazing incidence geometry.

\begin{tabular}{ccccc}
\hline Analyzer & Brewster Angle & $\boldsymbol{R}_{\boldsymbol{s}}(\boldsymbol{\%})$ & $\boldsymbol{R}_{\boldsymbol{p}}(\boldsymbol{\%})$ & $\boldsymbol{P}$ \\
\hline ML for $150 \mathrm{eV}$ & $45^{\circ}$ & 7.0 & 0.021 & 0.994 \\
Au miror for $56 \mathrm{eV}$ & $49^{\circ}$ & 5.5 & 0.28 & 0.903 \\
\hline
\end{tabular}

The XUV intensity recorded with the XUV photo diode is a function of the azimuthal rotation angle, $\alpha$, and is given by [36]:

$$
I_{ \pm}=\frac{1}{2} I_{0} T\left(R_{s}+R_{p}\right)\left[1+P_{\operatorname{Lin}} P \cos \left(2 \alpha \pm 2 \theta_{F}\right)\right],
$$

where $I_{0}$ is the incident intensity, $T$ the transmittance of the sample and $P_{\text {Lin }}$ takes into account the small effects due to an induced ellipticity of the beam after transmission through the magnetized sample.

In Figure 3a, we show the measured intensity for photon energies resonant at the Gd $N_{4,5}$ edge. The measurements before optical excitation at $t=-10 \mathrm{ps}$ for two opposite magnetization directions of the sample are shifted by $2 \theta_{F}=4^{\circ}$. We note that the rotation angle is smaller than the maximum value that is predicted according to the measurement shown in Figure 1. We attribute this to the uncertainty in determining the absolute photon energy combined with the difficulty to scan the photon energy 
with fixed undulator gaps at FLASH. The spectral bandwidth of the FEL radiation on the order of $1-2 \%$ also results in an effectively smaller value of $\theta_{F}$. At 3 ps after optical excitation, we completely quench the magnetization and, consequently, the two measurements for opposite magnetization directions lie on top of each other and are centered around $\alpha=0^{\circ}$. In Figure $3 \mathrm{~b}$, we show the magnetic asymmetry calculated as $A=\left(I_{+}-I_{-}\right) /\left(I_{+}+I_{-}\right)$. Before time delay zero, we observe a very large asymmetry of approximately $40 \%$ at a rotation angle of $\alpha= \pm 4^{\circ}$, while 3 ps after optical excitation, the asymmetry is almost completely zero for all angles $\alpha$.
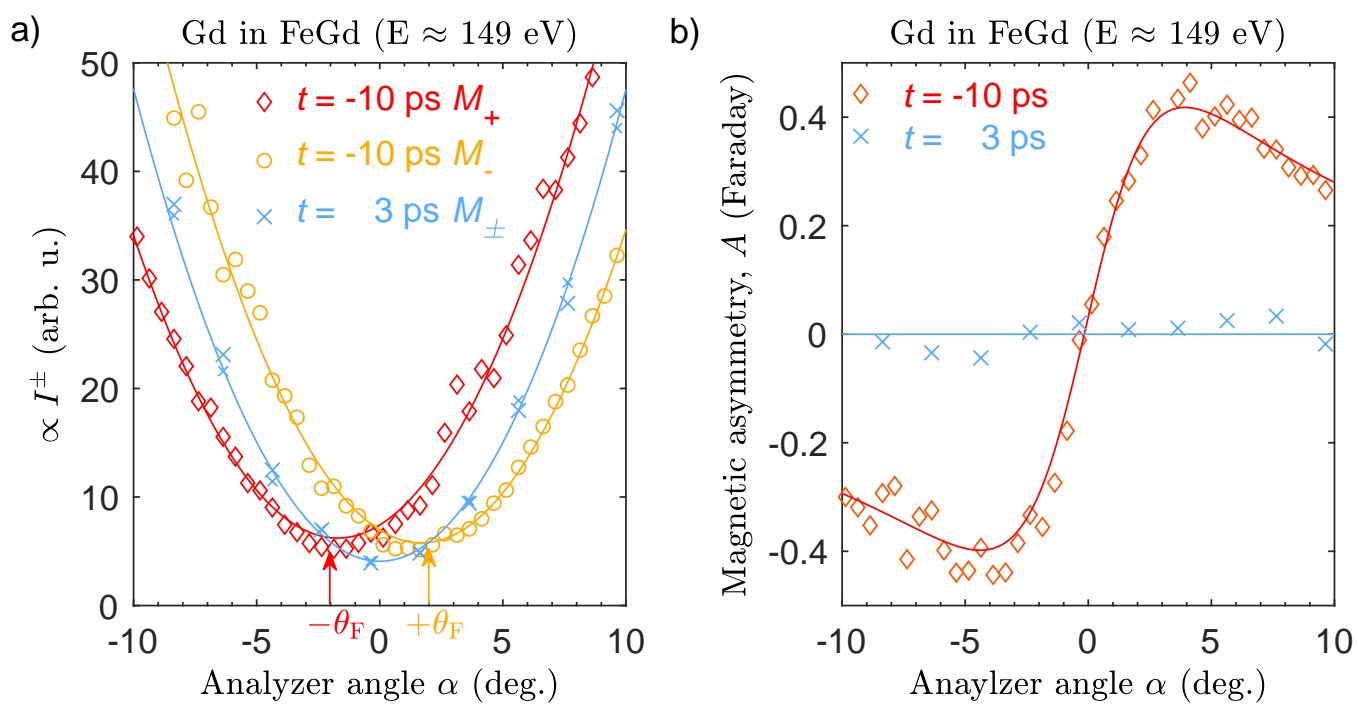

Figure 3. (a) Detected intensity after reflection off the analyzer mirror as a function of rotation angle, $\alpha$, for $E \approx 149 \mathrm{eV}$, resonant at the $N_{4,5}$ edge of $\mathrm{Gd}$. The two measurements before optical excitation at $t=-10 \mathrm{ps}$ for two opposite directions of the sample magnetization, $M_{ \pm}$, are shifted by the Faraday angle $2 \theta_{F}=4^{\circ}$. After optical excitation at $t=3 \mathrm{ps}$, the Faraday angle is zero, $\theta_{F}=0$, and the measurements are centered around $\alpha=0^{\circ}$, independent of the applied magnetic field. The lines are non-linear least square fits according to Equation (4). (b) Magnetic asymmetry before and after optical excitation is shown as a function of the analyzer angle. The maximum value reaches very large values of approximately $\pm 40 \%$ for $\pm \alpha= \pm 2 \theta_{F}= \pm 4^{\circ}$.

In Figure $4 \mathrm{a}$, we show the intensity $I_{ \pm}$measured at an angle of $\alpha=4^{\circ}$ as a function of the time delay between the optical excitation and the XUV probe pulses. Upon excitation, we observe an ultrafast drop or increase in the intensity depending on the magnetization direction of the sample. For the approximation that $\alpha \pm \theta_{F} \ll 1 \mathrm{rad}$ and for $P_{\operatorname{Lin}} \approx 1$, we can show that [37]:

$$
I_{+}-I_{-} \propto \theta_{F} \propto \Delta \delta \propto M .
$$

We normalize our observable, proportional to $M(t)$, to the average value before optical excitation, $M_{0}$, and show the corresponding relative changes in the magnetization as a function of time delay in Figure $4 \mathrm{~b}$. A nonlinear least square fit of the data with a mono-exponential function convolved with the temporal resolution of the experiment yields a demagnetization amplitude of the Gd sublattice by approximately $80 \%$ with a corresponding time constant of $\tau=(233 \pm 16)$ fs. Analogously measured time resolved data $M(t) / M_{0}$ at the Fe $M_{2,3}$ edge are shown in Figure 4c. While the amplitude of the demagnetization after $1 \mathrm{ps}$ is comparable between the $\mathrm{Fe}$ and $\mathrm{Gd}$ sublattices, in $\mathrm{Fe}$, we observe a significantly faster time constant of only $\tau=(94 \pm 16)$ fs. Distinct demagnetization times of the Gd and Fe sublattices in an FeGd alloy have been observed before in time resolved magnetic circular dichroism experiments. The microscopic origin has been explained by an ultrafast reduction in the interatomic exchange interaction due to the rapid heating of the electronic system close to, or even above the Curie 
temperature [10]. The demagnetization of the two sublattices is then allowed to proceed independently and scales with the very different magnetic moments of $\mathrm{Fe}\left(\mu_{\mathrm{at}} \approx 2.22 \mu_{\mathrm{B}}\right)$ and $\mathrm{Gd}\left(\mu_{\mathrm{at}} \approx 7.55 \mu_{\mathrm{B}}\right)$ [44]. As mentioned above, distinct sublattices with a different ultrafast magnetic response are a prerequisite for the formation of a transient ferromagnetic alignment of the two sublattices and, hence, for the occurrence of all-optical magnetic switching [45]. There are two more aspects which are noteworthy: first, as we qualitatively confirm the results of the FeGd demagnetization dynamics carried out with the established technique of MCD in the soft-X-ray range $[10,44,46]$, we conclude that ultrafast Faraday rotation is indeed an accurate observable for the transient magnetization. Second, a lot of research in resonant XUV spectroscopy has recently been devoted to exploring a potential influence of transient changes in the electro-optical functions $\beta(t)$ and $\delta(t)$ to MCD signals [26,29,34,47-49]. Transient changes in the transmission, $T$ [18], or reflection [50] at a core-valence band transition have been shown to be on the order of a few percent, and hence of a similar magnitude compared to the changes in MCD signals in the XUV spectral range [38]. This is different for the Faraday rotation. On the one hand, we observe very large asymmetries of up to $40 \%$ and, on the other hand, small relative changes in the transmission, $T$, have a negligible influence on $\left(I_{+}-I_{-}\right)$for $\alpha \ll 1$, as is easily verified by inspection of Equation (4). Therefore, non-equilibrium electron distributions are expected to have an insignificant effect on the extracted magnetization dynamics.
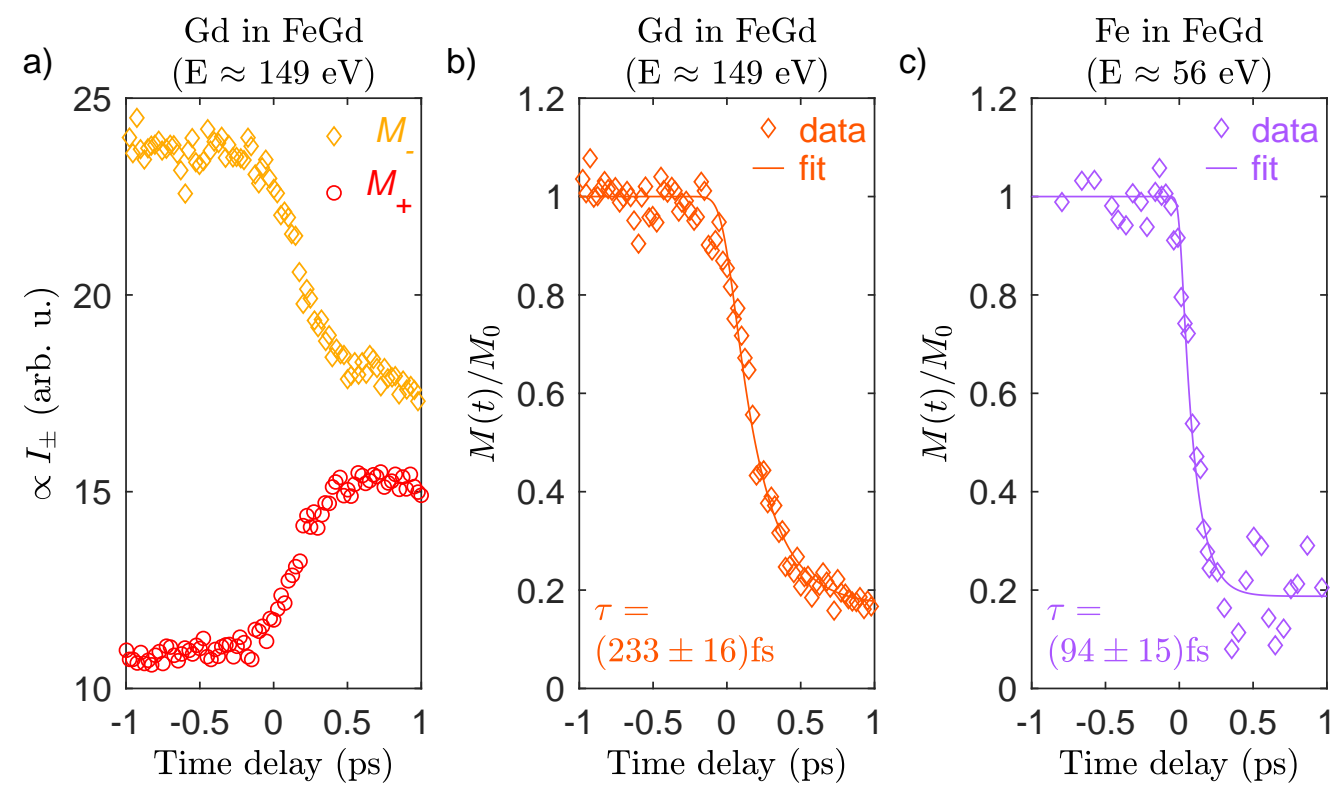

Figure 4. (a) Measurements of $I_{ \pm}$for opposite magnetization directions, $M_{ \pm}$, as a function of the time delay at the $\mathrm{Gd} N_{4,5}$ resonance at $E \approx 149 \mathrm{eV}$. Corresponding values of $M(t) / M_{0}$ (b) for the Gd and (c) Fe sublattice determined according to Equation (5). Solid lines are non-linear least-square fit with a monoexponential function with a time constant $\tau=(233 \pm 16)$ fs and $\tau=(94 \pm 15)$ fs for Gd and Fe, respectively.

\subsection{Time Resolved Magnetic Circular Dichroism and Helicity-Dependent Absorption}

In the second part of this paper, we present recent results on the hypothesis that tailored chemical heterogeneities in magnetic systems can be used to enhance the ultrafast magnetic response after optical excitation. As outlined in the introduction, in multi-component magnetic systems with sites exhibiting a distinct number of available states above the Fermi energy, OISTR can become an important mechanism for ultrafast demagnetization. To this end, we have compared the ultrafast magnetization dynamics of an elemental Co film and $\mathrm{Co}_{0.5} \mathrm{Pt}_{0.5}$ alloy via MCD in transmission geometry [18], as schematically depicted in Figure $2 b$. We generate high harmonic radiation by focusing intense laser pulses into a neon-filled gas cell, leading to discrete harmonic emission peaks in the spectral 
range between $\approx 40-72 \mathrm{eV}$ with a width of $\approx 200 \mathrm{meV}$. The state of polarization is controlled by a four-mirror phase-shifter [24,51,52], resulting in a high degree of circular polarization of $>80 \%$ in the energy range between 50 and $65 \mathrm{eV}$. The broadband XUV spectrum is transmitted through the sample, energetically dispersed and measured by a charged coupled device (CCD). As both magnetic films, $\mathrm{Co}$ and $\mathrm{CoPt}$, have their easy magnetization direction oriented in the sample plane (cf. schematic in Figure 2d,e), we mounted the sample at $\theta=45^{\circ}$ with respect to the incoming wave vector of the XUV light to ensure a finite projection onto the in-plane magnetization. The samples were excited in a collinear geometry with laser pulses centered at a wavelength of $\lambda=800 \mathrm{~nm}$, a fluence of $\approx 15 \mathrm{~mJ} \mathrm{~cm}{ }^{-2}$ and a pulse length of $39 \mathrm{fs}$. The experiment is carried out at a $3 \mathrm{kHz}$ repetition rate.

We measure the transmitted intensity, $I_{ \pm}\left(E, t, M_{ \pm}\right)$, for two opposite magnetization directions by toggling the external magnetic field, $B_{ \pm}$. Note that measurements with opposite magnetization directions of the sample but with a fixed helicity of the light is equivalent to measurements with opposite helicities but a fixed magnetization direction [19]. The magnetic asymmetry, $A$, is approximately proportional to the MCD contrast and is given by:

$$
A=\frac{I_{+}-I_{-}}{I_{+}+I_{-}} \approx \frac{1}{2} \ln \left(\frac{I_{+}}{I_{-}}\right)=-\frac{2 E d}{\hbar c} \Delta \beta \propto M .
$$

To increase the signal to noise ratio, we record the incoming spectrum with a second spectrometer and repeat the time delay scans up to 100 times. The total measurement time per sample amounts to about $4 \mathrm{~h}$ for each sample. For more details on the experimental details, we refer to Yao et al. [53].

In Figure 5, we show the magnetic asymmetry as a function of the time delay after optical excitation. The asymmetry for the elemental Co film measured at the Co $M_{2,3}$ edge is $A=-6.6 \%$, which drops with a time constant $\tau\left(\mathrm{Co}: \mathrm{CoM}_{2,3}\right)=(124 \pm 4)$ fs by $20 \%$. For the CoPt alloy, we follow the element-specific response by recording the respective sublattices at the Co $M_{2,3}$ edge as well as at the $\mathrm{Pt} \mathrm{O}_{3}$ and $\mathrm{N}_{7}$ edge independently in the same measurement. Here, the asymmetries are smaller and remain below $1 \%$. Note that the small asymmetries in XUV MCD compared to Faraday rotation measurements require a significantly better noise management. The rms value for $A$ before $t=0$ fs corresponds to $<3 \times 10^{-4}$, yielding an excellent signal to noise ratio of our data. Non-linear least square fits (solid lines) reveal an equivalent response with a demagnetization amplitude of $80 \%$ and time constants of $\tau\left(\mathrm{CoPt}: \mathrm{Co} \mathrm{M}_{2,3}\right)=(86 \pm 3) \mathrm{fs}, \tau\left(\mathrm{CoPt}: \mathrm{Pt} \mathrm{O}_{3}\right)=(84 \pm 7)$ fs, $\tau\left(\mathrm{CoPt}: \mathrm{Pt} N_{7}\right)=(92 \pm 11)$ fs. A priori, identical local demagnetization times for Co and Pt are an unexpected result, because it is generally assumed that an important process responsible for ultrafast demagnetization relies on spin-flip scattering processes, which are mediated by spin-orbit interaction. With an approximately 10-times larger spin-orbit coupling in Pt compared to Co, we would anticipate a larger demagnetization rate localized at the $\mathrm{Pt}$ sites. This is, however, not what we observe. Additionally, we recall that CoPt compared to Co exhibits a significantly higher demagnetization rate and efficiency. This strongly suggests that an additional demagnetization channel is active in the two-component alloy CoPt. In the following section, we will demonstrate that this process can be identified as OISTR.

In Figure 6, we show a calculation of the laser-driven spin-dependent $3 d$ electron occupation, $\Delta n_{\min / \text { maj }}(t)=n_{\min / \text { maj }}(t)-n_{\min / \text { maj }}(t<0)$ around the Fermi energy of Co in the CoPt alloy and of the elemental Co film. The simulations are performed using a fully non-collinear version of TD-DFT as implemented within the Elk code [54]. Upon laser pumping, electrons are excited to available states above the Fermi energy. Because the density of states of the spin-split $3 d$ band of Co is dominated by available minority states, minority electrons are reshuffled more efficiently. In elemental $\mathrm{Co}$, we observe a small loss of majority electrons (spin up) and a small increase in minority electrons (spin down), which we can attribute to spin-flips, leading to the observed loss of magnetization. However, much more prominent is the very large gain in minority electrons in CoPt above the Fermi energy, significantly exceeding the loss of minority electrons below the Fermi energy (red dashed line). It is worth also noting that this is much more pronounced compared to the minority response of elemental 
Co (blue dashed line). The additional minority electrons stem from the Pt sublattice and are transferred in an optical transition between the respective $d$ minority states.

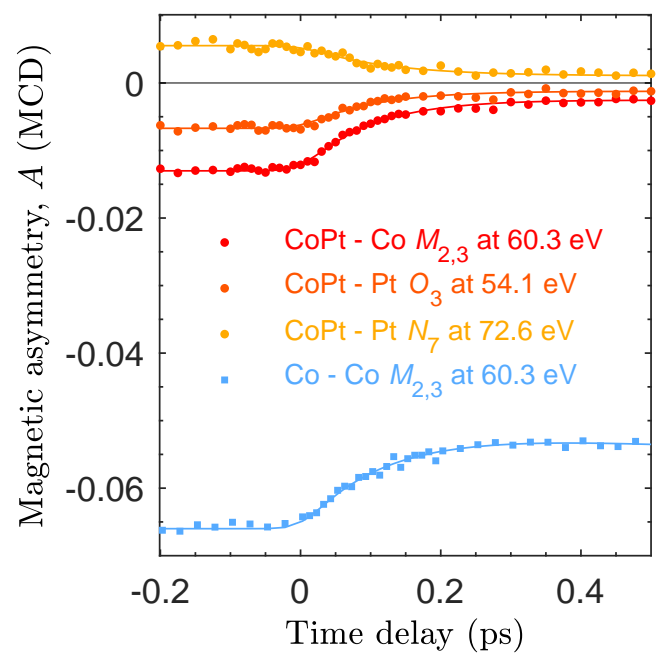

Figure 5. The magnetic asymmetry, $A(\mathrm{MCD})$, of the Co film and the CoPt alloy as a function of the time delay for three different photon energies corresponding to the Co $M_{2,3}$ edge at $60.3 \mathrm{eV}$ and to the $\mathrm{Pt} \mathrm{O}_{3}$ and $\mathrm{N}_{7}$ edge at $54.1 \mathrm{eV}$ and $72.6 \mathrm{eV}$, respectively. The lines are non-linear least square fits. The rms value for values before $t=0$ fs corresponds to $<3 \times 10^{-4}$ yielding an excellent signal to noise ratio in spite of small asymmetry values.
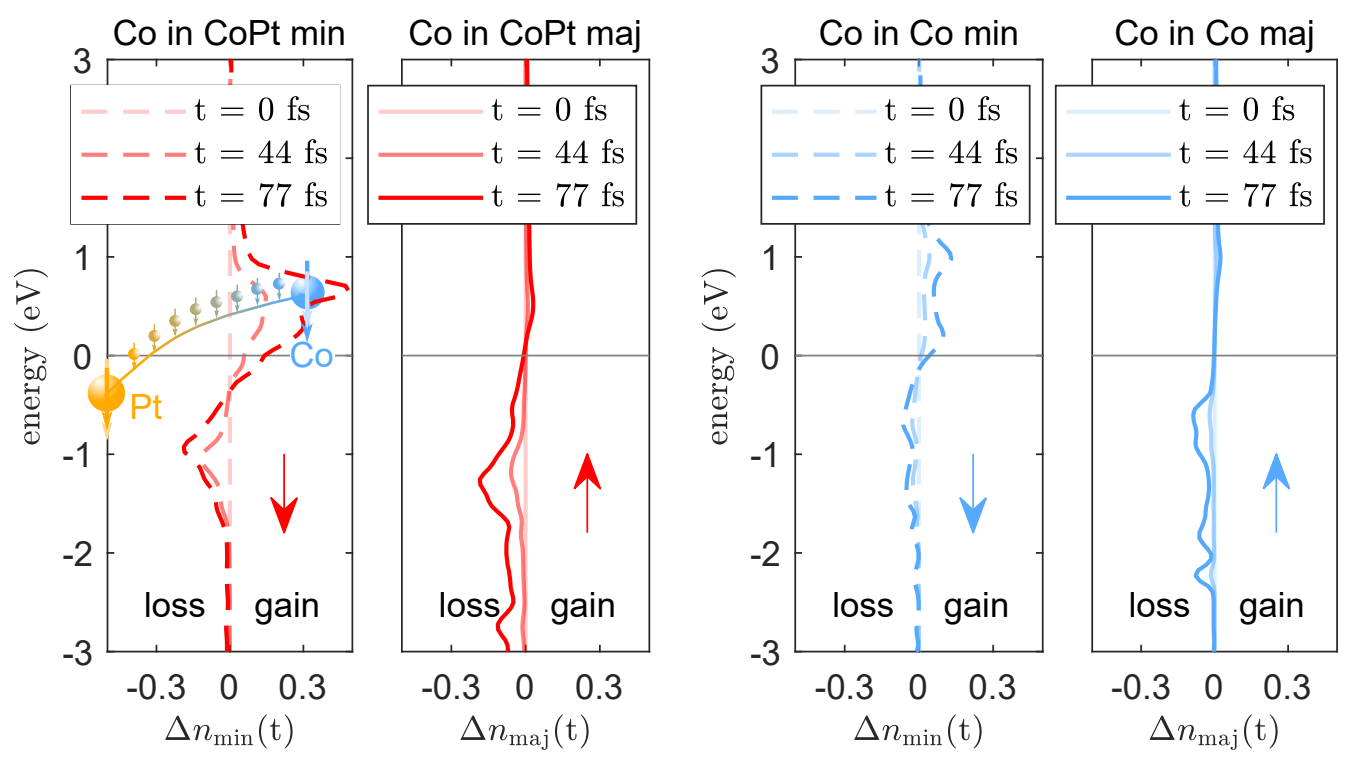

Figure 6. We show a calculation of the laser induced changes in the spin-dependent electron occupations in the $3 d$ band, $\Delta n_{\mathrm{min} / \mathrm{maj}}$ around the Fermi energy for Co in the CoPt alloy (red lines) and for elemental Co (blue lines). Laser excitation promotes electrons from occupied states below the Fermi energy to available states above the Fermi energy. Minority states (spin down) of Co in the CoPt alloy are filled much more efficiently than in the elemental $\mathrm{Co}$, indicating a transfer of minority states from $\mathrm{Pt} 5 d$ to Co $3 d$ states, significantly increasing the efficiency of the demagnetization dynamics. This is schematically indicated in the left panel with minority electrons transferred from Pt to Co.

To experimentally demonstrate that the minority $3 d$ states of $\mathrm{Co}$ in CoPt are indeed efficiently filled in an OISTR process, we examine the helicity-dependent absorption channels individually. We calculate the absorption as $\mu_{ \pm}=1-I_{ \pm}$, neglecting the small reflectance in the XUV spectral range. 
We show the normalized result in Figure 7 as a function of time delay between $-50 \mathrm{fs}$ and $100 \mathrm{fs}$ : in the left panels, we plot the experimental data for $\mathrm{Co}$ and $\mathrm{CoPt}$; in the right panels are the corresponding theoretically calculated values. Importantly, careful theoretical analysis shows that we can associate the transient absorption changes $\mu / \mu_{0}$ measured with opposite magnetization directions $M_{+}$and $M_{-}$ with transitions predominantly into the majority and minority states, respectively.
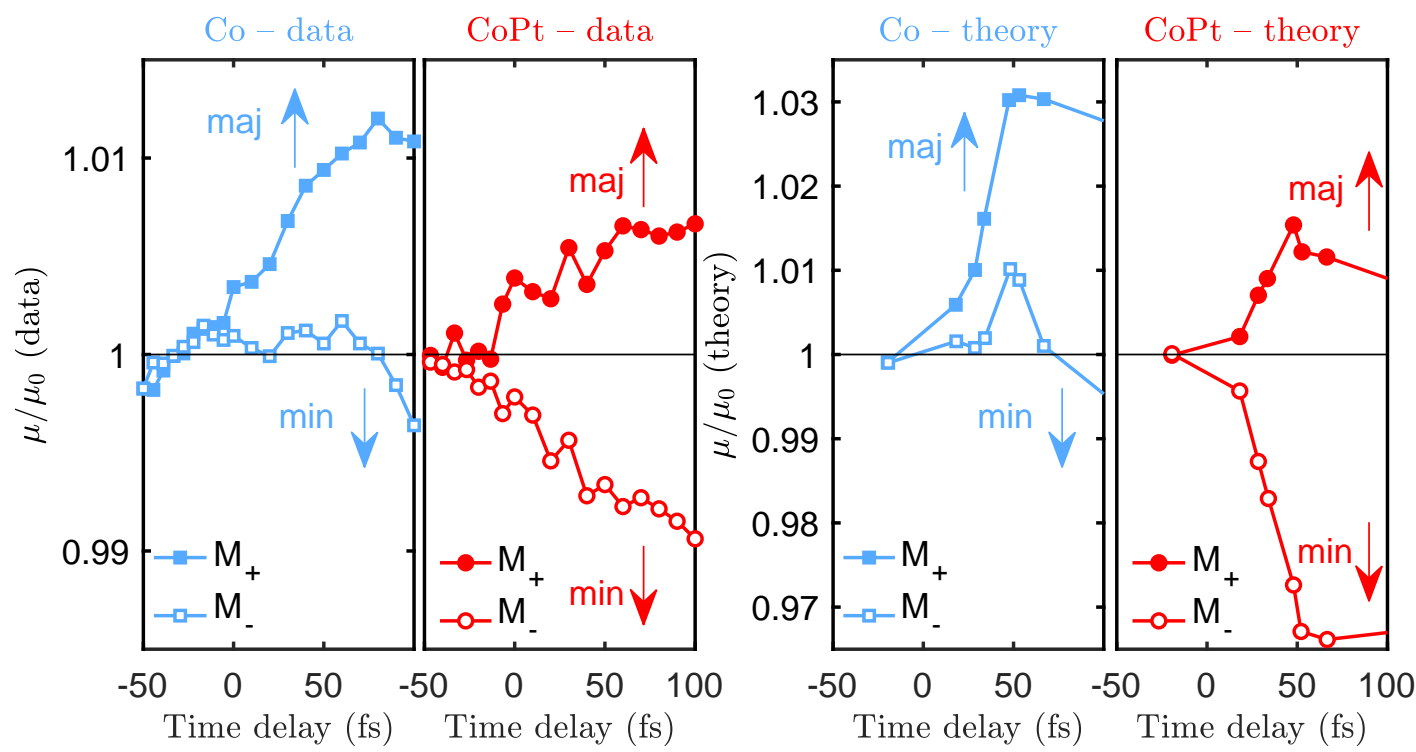

Figure 7. Normalized helicity-dependent absorption, $\mu / \mu_{0}$ as a function of time delay for Co (blue squares) and CoPt (red circles). In the left panels, we show data measured at the Co $M_{2,3}$ resonance at $60 \mathrm{eV}$; in the right panel are the corresponding calculations. A positive magnetic field, $M_{+}$, predominantly probes changes in majority occupations (spin up) and a negative magnetic field, $M_{-}$, predominantly probes changes of the minority occupations (spin down). In CoPt we can clearly see that the absorption into minority states very rapidly decreases, which we interpret as the efficient filling of minority states by OISTR-i.e., transitions of $\mathrm{Pt} 5 d$ to Co $3 d$ minority states.

The most apparent differences of $\mu / \mu_{0}$ between the elemental Co and CoPt alloy is the response of the absorption for a sample magnetized in $M_{-}$direction (open symbols in Figure 7): in Co, the absorption slightly increases or stays constant while in $\mathrm{CoPt}$ the absorption drops immediately after optical excitation. The interpretation is now straightforward and can be linked with the transient occupation changes, $\Delta n_{\mathrm{min}}$, shown in Figure 6: efficient filling of Co minority states above the Fermi energy by OISTR from $\mathrm{Pt}$ reduces the available final states for transitions, leading to an ultrafast drop of absorption. In elemental $\mathrm{Co}$, this is not observed, again consistent with the transient occupations shown in Figure 6. The theoretical calculation of the exact same observable, $\mu / \mu_{0}$, reproduces our experimental finding very well [18].

Finally, we remark that the distinct response between the absorption measured with different magnetization directions of the sample (or opposite helicities), as observed in the experiment, further strongly supports the theoretical finding that helicity-dependent absorption measures the spin-resolved transient changes of the occupations around the Fermi level of a specific element for early times after optical excitation $(\approx 100 \mathrm{fs})$.

\section{Conclusions}

As optically induced functionalities of magnetic materials are closely linked to the ultrafast interplay of different elements within a magnetic system, access to element-specific observables is a necessity for progress on the path from a fundamental understanding to potential applications. We have demonstrated 
that resonant XUV spectroscopy in conjunction with state-of-the-art theory is indeed capable of providing a detailed picture of the microscopic processes responsible for macroscopic magnetization.

We demonstrated this by two complementary experimental realizations probing either the dispersive or the absorptive part of the magneto-optical functions in a Faraday rotation and an MCD experiment, respectively. In the first experiment, the polarization plane of transmitted photons tuned to the respective core-to-valence band transition in an FeGd alloy yielded large Faraday rotation angles and allowed us to reveal decoupled ultrafast magnetization dynamics of the Gd and Fe sublattices. Three advantages of Faraday rotation measurements are noteworthy: first, they yield large magnetic asymmetries; second, they do not require polarization control of the XUV radiation, which has remained challenging in HHG and is not (yet) always available at free-electron laser facilities; finally, the intrinsic contrast of Faraday measurements is only based on a phase difference and is therefore not detrimentally affected by strong absorption losses at the resonance of the probed element.

In the second experiment, we employed MCD contrast to reveal an efficient inter-site spin transfer in the two-component $\mathrm{CoPt}$ alloy. As the strength and direction of such spin-selective charge flow is determined by the available states above the Fermi energy of the involved elements [55], we envisioned to tailor the ultrafast magnetic response and work towards realizing a metamagnetic phase transition on the fastest time scale. While MCD experiments in the XUV spectral range require circularly polarized radiation and are technically very demanding because of very small magnetic asymmetries, they offer the great potential to analyze the energy and helicity-dependent transient absorption in order to differentiate the ultrafast occupation changes of minority and majority electrons after optical excitation.

Finally, we remark that, with our recent successful efforts to extend the photon range of HHG sources beyond the water window [56], we expect, in the very near future, the first experiments with photon energies around the giant $N$ resonances of rare earth elements, as well as around $L$ resonances of transition metals in laboratory based experiments.

\section{Materials and Methods}

The thin magnetic films, $\mathrm{Co}, \mathrm{Co}_{0.5} \mathrm{Pt}_{0.5}$ and $\mathrm{Gd}_{0.24} \mathrm{Fe}_{0.76}$ were grown by $\mathrm{DC}$ magnetron sputtering. The FeGd alloy with a thickness of $15 \mathrm{~nm}$ was grown on a free standing DMC (diamond like carbon) membrane. Its magnetization pointed out of the sample plane with a coercive field of $50 \mathrm{mT}$. The $\mathrm{Co}$ and CoPt films with a thickness of $15 \mathrm{~nm}$ were deposited on freestanding $20 \mathrm{~nm}$ thick $\mathrm{Si}_{3} \mathrm{~N}_{4}$ and $200 \mathrm{~nm}$ thick Al membranes, respectively. They exhibit an in-plane anisotropy and were consequently mounted under a grazing angle of 45 degrees for a finite projection of the k-vector of the circularly polarized XUV pulses onto the magnetization of the sample. They exhibit a square hysteresis loop with a coercive field below $10 \mathrm{mT}$.

Author Contributions: C.v.K.S., S.E., F.W., I.R. and S.S. conceived the experiments, C.v.K.S., M.H., I.R., C.S., V.S., J.B., G.H., K.B., J.V., B.M., S.G., S.D., E.J., B.V. and J.L. performed the experiment at FLASH. J.L. programmed and implemented the data acquisition software for the FLASH experiment. F.W., K.Y., D.S., C.S. and C.v.K.S. performed the HHG experiments. D.S. programmed and implemented the data acquisition software for the HHG experiment. S.S. performed the $a b$ initio calculations. D.W.E. grew the samples. C.v.K.S., F.W., S.S., S.E., K.Y., M.B. and I.R. interpreted and discussed the results. C.v.K.S. prepared the figures and wrote the manuscript. All authors have read and agreed to the published version of the manuscript.

Funding: This research was funded by DFG through TRR227 project A02 and A04. The authors are grateful for financial support of the CNRS-PICS program.

Acknowledgments: We kindly acknowledge the invaluable help by the different support groups at FLASH, Desy in Hamburg.

Conflicts of Interest: The authors declare no conflict of interest. The funders had no role in the design of the study; in the collection, analyses, or interpretation of data; in the writing of the manuscript, or in the decision to publish the results. 


\section{Abbreviations}

The following abbreviations are used in this manuscript:

CCD charged coupled device

FEL free electron laser

HHG high harmonic generation

MCD magnetic circular dichroism

OISTR optical inter-site spin transfer

XUV extreme ultraviolet

\section{References}

1. Beaurepaire, E.; Merle, J.C.; Daunois, A.; Bigot, J.Y. Ultrafast Spin Dynamics in Ferromagnetic Nickel. Phys. Rev. Lett. 1996, 76, 4250-4253. [CrossRef] [PubMed]

2. Kirilyuk, A.; Kimel, A.V.; Rasing, T. Ultrafast optical manipulation of magnetic order. Rev. Mod. Phys. 2010, 82, 2731-2784. [CrossRef]

3. Koopmans, B.; Malinowski, G.; Dalla Longa, F.; Steiauf, D.; Fähnle, M.; Roth, T.; Cinchetti, M.; Aeschlimann, M. Explaining the paradoxical diversity of ultrafast laser-induced demagnetization. Nat. Mater. 2010, 9, 259-265. [CrossRef]

4. Schmidt, A.B.; Pickel, M.; Donath, M.; Buczek, P.; Ernst, A.; Zhukov, V.P.; Echenique, P.M.; Sandratskii, L.M.; Chulkov, E.V.; Weinelt, M. Ultrafast Magnon Generation in an Fe Film on Cu(100). Phys. Rev. Lett. 2010, 105, 197401. [CrossRef]

5. Carpene, E.; Hedayat, H.; Boschini, F.; Dallera, C. Ultrafast demagnetization of metals: Collapsed exchange versus collective excitations. Phys. Rev. B 2015, 91, 174414. [CrossRef]

6. $\quad$ Eich, S.; Plötzing, M.; Rollinger, M.; Emmerich, S.; Adam, R.; Chen, C.; Kapteyn, H.C.; Murnane, M.M.; Plucinski, L.; Steil, D.; et al. Band structure evolution during the ultrafast ferromagnetic-paramagnetic phase transition in cobalt. Sci. Adv. 2017, 3, e1602094. [CrossRef] [PubMed]

7. Malinowski, G.; Dalla Longa, F.; Rietjens, J.H.H.; Paluskar, P.V.; Huijink, R.; Swagten, H.J.M.; Koopmans, B. Control of speed and efficiency of ultrafast demagnetization by direct transfer of spin angular momentum. Nat. Phys. 2008, 4, 855-858. [CrossRef]

8. Battiato, M.; Carva, K.; Oppeneer, P.M. Superdiffusive Spin Transport as a Mechanism of Ultrafast Demagnetization. Phys. Rev. Lett. 2010, 105, 027203. [CrossRef]

9. Stanciu, C.D.; Tsukamoto, A.; Kimel, A.V.; Hansteen, F.; Kirilyuk, A.; Itoh, A.; Rasing, T. Subpicosecond Magnetization Reversal across Ferrimagnetic Compensation Points. Phys. Rev. Lett. 2007, 99, 217204. [CrossRef] [PubMed]

10. Radu, I.; Vahaplar, K.; Stamm, C.; Kachel, T.; Pontius, N.; Dürr, H.A.; Ostler, T.A.; Barker, J.; Evans, R.F.L.; Chantrell, R.W.; et al. Transient ferromagnetic-like state mediating ultrafast reversal of antiferromagnetically coupled spins. Nature 2011, 472, 205-208. [CrossRef] [PubMed]

11. Ostler, T.; Barker, J.; Evans, R.; Chantrell, R.; Atxitia, U.; Chubykalo-Fesenko, O.; El Moussaoui, S.; Le Guyader, L.; Mengotti, E.; Heyderman, L.; et al. Ultrafast heating as a sufficient stimulus for magnetization reversal in a ferrimagnet. Nat. Commun. 2012, 3, 666. [CrossRef] [PubMed]

12. Dewhurst, J.K.; Elliott, P.; Shallcross, S.; Gross, E.K.U.; Sharma, S. Laser-Induced intersite Spin Transfer. Nano Lett. 2018, 18, 1842-1848. [CrossRef] [PubMed]

13. Chen, J.; Bovensiepen, U.; Eschenlohr, A.; Müller, T.; Elliott, P.; Gross, E.K.U.; Dewhurst, J.K.; Sharma, S. Competing Spin Transfer and Dissipation at $\mathrm{Co} / \mathrm{Cu}(001)$ Interfaces on Femtosecond Timescales. Phys. Rev. Lett. 2019, 122, 067202. [CrossRef]

14. Steil, D.; Walowski, J.; Gerhard, F.; Kiessling, T.; Ebke, D.; Thomas, A.; Kubota, T.; Oogane, M.; Ando, Y.; Otto, J.; et al. Efficiency of ultrafast optically induced spin transfer in Heusler compounds. Phys. Rev. Res. 2020, 2, 023199. [CrossRef]

15. Siegrist, F.; Gessner, J.A.; Ossiander, M.; Denker, C.; Chang, Y.P.; Schröder, M.C.; Guggenmos, A.; Cui, Y.; Walowski, J.; Martens, U.; et al. Light-wave dynamic control of magnetism. Nature 2019, 571, $240-244$. [CrossRef] [PubMed] 
16. Tengdin, P.; Gentry, C.; Blonsky, A.; Zusin, D.; Gerrity, M.; Hellbrück, L.; Hofherr, M.; Shaw, J.; Kvashnin, Y.; Delczeg-Czirjak, E.K.; et al. Direct light-induced spin transfer between different elements in a spintronic Heusler material via femtosecond laser excitation. Sci. Adv. 2020, 6, eaaz1100. [CrossRef]

17. Hofherr, M.; Häuser, S.; Dewhurst, J.K.; Tengdin, P.; Sakshath, S.; Nembach, H.T.; Weber, S.T.; Shaw, J.M.; Silva, T.J.; Kapteyn, H.C.; et al. Ultrafast optically induced spin transfer in ferromagnetic alloys. Sci. Adv. 2020, 6, eaay8717. [CrossRef]

18. Willems, F.; von Korff Schmising, C.; Strüber, C.; Schick, D.; Engel, D.W.; Dewhurst, J.K.; Elliott, P.; Sharma, S.; Eisebitt, S. Optical inter-site spin transfer probed by energy and spin-resolved transient absorption spectroscopy. Nat. Commun. 2020, 11, 871. [CrossRef]

19. Stöhr, J.; Siegmann, H.C. Magnetism: From Fundamentals to Nanoscale Dynamics; Springer: Berlin/Heidelberg, Germany, 2006; Volume 152, pp. 1-822. [CrossRef]

20. Stamm, C.; Kachel, T.; Pontius, N.; Mitzner, R.; Quast, T.; Holldack, K.; Khan, S.; Lupulescu, C.; Aziz, E.F.; Wietstruk, M.; et al. Femtosecond modification of electron localization and transfer of angular momentum in nickel. Nat. Mater. 2007, 6, 740-743. [CrossRef]

21. Gutt, C.; Streit-Nierobisch, S.; Stadler, L.M.; Pfau, B.; Günther, C.M.; Könnecke, R.; Frömter, R.; Kobs, A.; Stickler, D.; Oepen, H.P.; et al. Single-pulse resonant magnetic scattering using a soft x-ray free-electron laser. Phys. Rev. B 2010, 81, 100401. [CrossRef]

22. La-O-Vorakiat, C.; Siemens, M.; Murnane, M.M.; Kapteyn, H.C.; Mathias, S.; Aeschlimann, M.; Grychtol, P.; Adam, R.; Schneider, C.M.; Shaw, J.M.; et al. Ultrafast Demagnetization Dynamics at the M Edges of Magnetic Elements Observed Using a Tabletop High-Harmonic Soft X-Ray Source. Phys. Rev. Lett. 2009, 103, 257402. [CrossRef] [PubMed]

23. Mathias, S.; La-O-Vorakiat, C.; Grychtol, P.; Granitzka, P.; Turgut, E.; Shaw, J.M.; Adam, R.; Nembach, H.T.; Siemens, M.E.; Eich, S.; et al. Probing the timescale of the exchange interaction in a ferromagnetic alloy. Proc. Natl. Acad. Sci. USA 2012, 109, 4792-4797. [CrossRef] [PubMed]

24. Willems, F.; Smeenk, C.T.L.; Zhavoronkov, N.; Kornilov, O.; Radu, I.; Schmidbauer, M.; Hanke, M.; von Korff Schmising, C.; Vrakking, M.J.J.; Eisebitt, S. Probing ultrafast spin dynamics with high-harmonic magnetic circular dichroism spectroscopy. Phys. Rev. B 2015, 92, 220405. [CrossRef]

25. Rudolf, D.; La-O-Vorakiat, C.; Battiato, M.; Adam, R.; Shaw, J.M.; Turgut, E.; Maldonado, P.; Mathias, S.; Grychtol, P.; Nembach, H.T.; et al. Ultrafast magnetization enhancement in metallic multilayers driven by superdiffusive spin current. Nat. Commun. 2012, 3, 1037. [CrossRef] [PubMed]

26. Turgut, E.; La-o vorakiat, C.; Shaw, J.M.; Grychtol, P.; Nembach, H.T.; Rudolf, D.; Adam, R.; Aeschlimann, M.; Schneider, C.M.; Silva, T.J.; et al. Controlling the Competition between Optically Induced Ultrafast Spin-Flip Scattering and Spin Transport in Magnetic Multilayers. Phys. Rev. Lett. 2013, 110, 197201. [CrossRef]

27. Vodungbo, B.; Gautier, J.; Lambert, G.; Sardinha, A.B.; Lozano, M.; Sebban, S.; Ducousso, M.; Boutu, W.; Li, K.; Tudu, B.; et al. Laser-induced ultrafast demagnetization in the presence of a nanoscale magnetic domain network. Nat. Commun. 2012, 3, 999. [CrossRef] [PubMed]

28. Pfau, B.; Schaffert, S.; Müller, L.; Gutt, C.; Al-Shemmary, A.; Büttner, F.; Delaunay, R.; Düsterer, S.; Flewett, S.; Frömter, R.; et al. Ultrafast optical demagnetization manipulates nanoscale spin structure in domain walls. Nat. Commun. 2012, 3, 1100. [CrossRef]

29. Weder, D.; von Korff Schmising, C.; Günther, C.M.; Schneider, M.; Engel, D.; Hessing, P.; Strüber, C.; Weigand, M.; Vodungbo, B.; Jal, E.; et al. Transient magnetic gratings on the nanometer scale. Struct. Dyn. 2020, 7, 054501. [CrossRef] [PubMed]

30. Schneider, M.; Pfau, B.; Günther, C.M.; von Korff Schmising, C.; Weder, D.; Geilhufe, J.; Perron, J.; Capotondi, F.; Pedersoli, E.; Manfredda, M.; et al. Ultrafast Demagnetization Dominates Fluence Dependence of Magnetic Scattering at Co M Edges. Phys. Rev. Lett. 2020, 125, 127201. [CrossRef]

31. von Korff Schmising, C.; Pfau, B.; Schneider, M.; Günther, C.M.; Giovannella, M.; Perron, J.; Vodungbo, B.; Müller, L.; Capotondi, F.; Pedersoli, E.; et al. Imaging Ultrafast Demagnetization Dynamics after a Spatially Localized Optical Excitation. Phys. Rev. Lett. 2014, 112, 217203. [CrossRef]

32. Turgut, E.; Zusin, D.; Legut, D.; Carva, K.; Knut, R.; Shaw, J.M.; Chen, C.; Tao, Z.; Nembach, H.T.; Silva, T.J.; et al. Stoner versus Heisenberg: Ultrafast exchange reduction and magnon generation during laser-induced demagnetization. Phys. Rev. B 2016, 94, 1-6. [CrossRef] 
33. Zusin, D.; Tengdin, P.M.; Gopalakrishnan, M.; Gentry, C.; Blonsky, A.; Gerrity, M.; Legut, D.; Shaw, J.M.; Nembach, H.T.; Silva, T.J.; et al. Direct measurement of the static and transient magneto-optical permittivity of cobalt across the entire M-edge in reflection geometry by use of polarization scanning. Phys. Rev. B 2018, 97, 024433. [CrossRef]

34. Jana, S.; Malik, R.S.; Kvashnin, Y.O.; Locht, I.L.M.; Knut, R.; Stefanuik, R.; Di Marco, I.; Yaresko, A.N.; Ahlberg, M.; Åkerman, J.; et al. Analysis of the linear relationship between asymmetry and magnetic moment at the M edge of $3 \mathrm{~d}$ transition metals. Phys. Rev. Res. 2020, 2, 013180. [CrossRef]

35. Kuneš, J.; Oppeneer, P.M.; Mertins, H.C.; Schäfers, F.; Gaupp, A.; Gudat, W.; Novák, P. X-ray Faraday effect at the L2,3 edges of Fe, Co, and Ni: Theory and experiment. Phys. Rev. B Condens. Matter Mater. Phys. 2001, 64, 1-10. [CrossRef]

36. Valencia, S.; Gaupp, A.; Gudat, W.; Mertins, H.C.; Oppeneer, P.M.; Abramsohn, D.; Schneider, C.M. Faraday rotation spectra at shallow core levels: 3p edges of Fe, Co, and Ni. New J. Phys. 2006, 8. [CrossRef]

37. Alves, C.; Lambert, G.; Malka, V.; Hehn, M.; Malinowski, G.; Hennes, M.; Chardonnet, V.; Jal, E.; Lüning, J.; Vodungbo, B. Resonant Faraday effect using high-order harmonics for the investigation of ultrafast demagnetization. Phys. Rev. B 2019, 100, 144421. [CrossRef]

38. Willems, F.; Sharma, S.; v. Korff Schmising, C.; Dewhurst, J.K.; Salemi, L.; Schick, D.; Hessing, P.; Strüber, C.; Engel, W.D.; Eisebitt, S. Magneto-Optical Functions at the 3 p Resonances of Fe, Co, and Ni: Ab initio Description and Experiment. Phys. Rev. Lett. 2019, 122, 217202. [CrossRef]

39. Willems, F.; von Korff Schmising, C.; Weder, D.; Günther, C.M.; Schneider, M.; Pfau, B.; Meise, S.; Guehrs, E.; Geilhufe, J.; Merhe, A.E.D.; et al. Multi-color imaging of magnetic Co/Pt heterostructures. Struct. Dyn. 2017, 4, 014301. [CrossRef]

40. Dewhurst, J.K.; Willems, F.; Elliott, P.; Li, Q.Z.; Schmising, C.V.K.; Strüber, C.; Engel, D.W.; Eisebitt, S.; Sharma, S. Element Specificity of Transient Extreme Ultraviolet Magnetic Dichroism. Phys. Rev. Lett. 2020, 124, 077203. [CrossRef]

41. Tiedtke, K.; Azima, A.; von Bargen, N.; Bittner, L.; Bonfigt, S.; Düsterer, S.; Faatz, B.; Frühling, U.; Gensch, M.; Gerth, C.; et al. The soft x-ray free-electron laser FLASH at DESY: Beamlines, diagnostics and end-stations. New J. Phys. 2009, 11, 023029. [CrossRef]

42. Rabinovitch, K.; Canfield, L.R.; Madden, R.P. A Method for Measuring Polarization in the Vacuum Ultraviolet. Appl. Opt. 1965, 4, 1005. [CrossRef]

43. Henke, B.; Gullikson, E.; Davis, J. X-Ray Interactions: Photoabsorption, Scattering, Transmission, and Reflection at $\mathrm{E}=50-30,000 \mathrm{eV}, \mathrm{Z}=1$-92. At. Data Nucl. Data Tables 1993, 54, 181-342. [CrossRef]

44. Radu, I.; Stamm, C.; Eschenlohr, A.; Radu, F.; Abrudan, R.; Vahaplar, K.; Kachel, T.; Pontius, N.; Mitzner, R.; Holldack, K.; et al. Ultrafast and Distinct Spin Dynamics in Magnetic Alloys. Spin 2015, 5, 1-10. [CrossRef]

45. Davies, C.; Janssen, T.; Mentink, J.; Tsukamoto, A.; Kimel, A.; van der Meer, A.; Stupakiewicz, A.; Kirilyuk, A. Pathways for Single-Shot All-Optical Switching of Magnetization in Ferrimagnets. Phys. Rev. Appl. 2020, 13, 024064. [CrossRef]

46. Hennecke, M.; Radu, I.; Abrudan, R.; Kachel, T.; Holldack, K.; Mitzner, R.; Tsukamoto, A.; Eisebitt, S. Angular Momentum Flow during Ultrafast Demagnetization of a Ferrimagnet. Phys. Rev. Lett. 2019, 122, 1-10. [CrossRef] [PubMed]

47. La-O-Vorakiat, C.; Turgut, E.; Teale, C.A.; Kapteyn, H.C.; Murnane, M.M.; Mathias, S.; Aeschlimann, M.; Schneider, C.M.; Shaw, J.M.; Nembach, H.T.; et al. Ultrafast Demagnetization Measurements Using Extreme Ultraviolet Light: Comparison of Electronic and Magnetic Contributions. Phys. Rev. X 2012, 2, 011005. [CrossRef]

48. Vodungbo, B.; Gautier, J.; Lambert, G.; Zeitoun, P.; Lüning, J. Comment on "Ultrafast Demagnetization Measurements Using Extreme Ultraviolet Light: Comparison of Electronic and Magnetic Contributions". Phys. Rev. X 2013, 3, 038001. [CrossRef]

49. Yao, K.; Willems, F.; von Korff Schmising, C.; Radu, I.; Strüber, C.; Schick, D.; Engel, D.; Tsukamoto, A.; Dewhurst, J.K.; Sharma, S.; et al. Distinct spectral response in M-edge magnetic circular dichroism. Phys. Rev. B 2020, 102, 100405. [CrossRef]

50. Tengdin, P.; You, W.; Chen, C.; Shi, X.; Zusin, D.; Zhang, Y.; Gentry, C.; Blonsky, A.; Keller, M.; Oppeneer, P.M.; et al. Critical behavior within 20 fs drives the out-of-equilibrium laser-induced magnetic phase transition in nickel. Sci. Adv. 2018, 4, 1-9. [CrossRef] 
51. Vodungbo, B.; Barszczak Sardinha, A.; Gautier, J.; Lambert, G.; Valentin, C.; Lozano, M.; Iaquaniello, G.; Delmotte, F.; Sebban, S.; Lüning, J.; et al. Polarization control of high order harmonics in the EUV photon energy range. Opt. Express 2011, 19, 4346. [CrossRef]

52. von Korff Schmising, C.; Weder, D.; Noll, T.; Pfau, B.; Hennecke, M.; Strüber, C.; Radu, I.; Schneider, M.; Staeck, S.; Günther, C.M.; et al. Generating circularly polarized radiation in the extreme ultraviolet spectral range at the free-electron laser FLASH. Rev. Sci. Instrum. 2017, 88, 053903. [CrossRef]

53. Yao, K.; Willems, F.; Schmising, C.V.K.; Engel, D.; Strüber, C.; Hessing, P.; Pfau, B.; Schick, D.; Eisebitt, S.; Schneider, M. A tabletop setup for ultrafast helicity- dependent and element-specific absorption spectroscopy and scattering in the extreme ultraviolet spectral range A tabletop setup for ultrafast helicity-dependent and element-specific absorption spectroscopy and scattering in the extreme ultraviolet spectral range. Rev. Sci. Instrum. 2020, 91, 093001. [CrossRef]

54. Dewhurst, J.K. The Elk Code 2018. Available online: http://elk.sourceforge.net/ (accessed on 26 October 2020.

55. Borchert, M.; Schmising, C.v.K.; Schick, D.; Engel, D.; Sharma, S.; Eisebitt, S. Manipulation of Ultrafast Demagnetization Dynamics by Optically Induced intersite Spin Transfer in Magnetic Compounds with Distinct Density of States. 2020. pp. 1-10. Available online: https:/ / arxiv.org/abs/2008.12612 (accessed on 26 October 2020).

56. Feng, T.; Heilmann, A.; Bock, M.; Ehrentraut, L.; Witting, T.; Yu, H.; Stiel, H.; Eisebitt, S.; Schnürer, M. 27 W $21 \mu \mathrm{m}$ OPCPA system for coherent soft X-ray generation operating at $10 \mathrm{kHz}$. Opt. Express 2020, $28,8724$. [CrossRef] [PubMed]

Publisher's Note: MDPI stays neutral with regard to jurisdictional claims in published maps and institutional affiliations.

(C) 2020 by the authors. Licensee MDPI, Basel, Switzerland. This article is an open access article distributed under the terms and conditions of the Creative Commons Attribution (CC BY) license (http:/ / creativecommons.org/licenses/by/4.0/). 